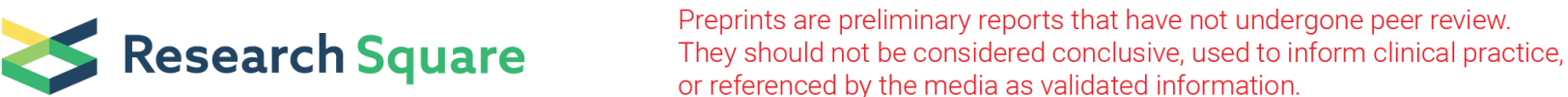

\section{ApoE and apoC-III-defined HDL subtypes: A descriptive study of their LCAT and CETP content and activity}

Mateo Amaya-Montoya

Universidad de Los Andes Facultad de Medicina

Jairo A Pinzón-Cortés

Universidad de los Andes Facultad de Medicina

Lina S Silva-Bermúdez

Universidad de los Andes Facultad de Medicina

Daniel Ruiz-Manco

Universidad de los Andes Facultad de Medicina

Maria C Perez-Matos

Universidad de los Andes Facultad de Medicina

Mario A Jimenez-Mora

Universidad de los Andes Facultad de Medicina

Carlos 0 Mendivil ( $\nabla$ carlosolimpo@gmail.com )

Universidad de los Andes https://orcid.org/0000-0001-5546-4206

\section{Research}

Keywords: HDL, lecithin cholesterol acyltransferase, cholesterol ester transfer protein, apolipoprotein $\mathrm{E}$, apolipoprotein C-III, reverse cholesterol transport

Posted Date: April 17th, 2020

DOI: https://doi.org/10.21203/rs.2.20333/v2

License: (c) (1) This work is licensed under a Creative Commons Attribution 4.0 International License.

Read Full License

Version of Record: A version of this preprint was published at Lipids in Health and Disease on May 25th, 2020. See the published version at https://doi.org/10.1186/s12944-020-01291-x. 


\section{Abstract}

Background High-density lipoproteins (HDL) functionality predicts cardiovascular risk better than HDL concentrations. The apolipoprotein composition of HDL may be a determinant of their function. Lecithincholesterol acyl transferase (LCAT) and cholesterol-ester transfer protein (CETP) are key enzymes for HDL-mediated reverse cholesterol transport. We assessed the distribution and activity of LCAT and CETP in HDL subspecies defined by their content of apolipoproteins $E$ (apoE) and C-III (apoC-III) in humans.

Methods We isolated in 18 adult humans of both sexes (mean age 55.6, BMI $26.9 \mathrm{Kg} / \mathrm{m} 2, \mathrm{HbA} 1 \mathrm{c} 5.4 \%$ ), four subspecies of HDL containing respectively: No apoE and no apoC-III (E-C-), apoE but not apoC-III $(\mathrm{E}+\mathrm{C}-)$, apoC-III but no apoE (E-C+) and both apoE and apoC-III (E+C+). In each HDL subspecies, we measured LCAT and CETP concentration and activity using immunoenzymatic and fluorometric methods. Additionally, we determined the size distribution of HDL in each apolipoprotein-defined fraction using non-denaturing electrophoresis and anti-ApoA-I western blot.

Results Similar to previous studies, HDL in the E-C- fraction was the predominant subtype. The size distribution of HDL was very similar across all four apolipoprotein-defined fractions. LCAT was most abundant in E-C- HDL (3.58 mg/mL, $59.6 \%$ of plasma LCAT mass), while HDL with apoE or apoC-III had much less LCAT $(19.8 \%, 12.2 \%$ and $8.37 \%$ of plasma LCAT respectively for E+C-, E-C+ and E+C+). LCAT mass was lower in E+C- HDL relative to E-C- HDL, but LCAT activity was similar in both fractions, signaling a greater activity-to-mass ratio associated with the presence of apoE. Both CETP mass and CETP activity showed only slight variations across HDL subspecies. There was an inverse correlation between plasma LCAT activity and both $\mathrm{E}-\mathrm{C}+$ pre-beta $\mathrm{HDL}(\mathrm{r}=-0.55, \mathrm{p}=0.017)$ and $\mathrm{E}-\mathrm{C}$ - alpha $1 \mathrm{HDL}$ $(r=-0.49, p=0.041)$. Conversely, there was a direct correlation between $\mathrm{E}-\mathrm{C}+$ alpha $1 \mathrm{HDL}$ and CETP activity in plasma $(r=0.52, p=0.025)$.

Conclusions Our results suggest that LCAT activity in humans is influenced by the presence of small interchangeable apolipoproteins. The presence of apoE in small HDL is correlated with increased LCAT activity and esterification of plasma cholesterol.

\section{Background}

A host of observational studies has demonstrated a negative association between plasma concentrations of high-density lipoprotein cholesterol (HDL-C) and the risk of cardiovascular disease (CVD) (1-4). However, medications aimed at raising HDL-C have failed to reduce the incidence of CVD in clinical trials. The groups of HDL-C raising agents that have proven ineffective in preventing CVD includes cholesteryl ester transfer protein (CETP) inhibitors, fibrates and niacin (5-8). This apparent paradox seems to be explained by the fact that HDL functionality, rather than HDL-C concentration, is the relevant measure associated with CVD prevention (9-10). Many aspects of HDL functionality have been evaluated over the recent years, including but not limited to reverse cholesterol transport (RCT), induction of nitric oxide synthesis, reduction in the expression of cell adhesion molecules and antioxidant capacity (11). 
Lecithin-cholesterol acyl transferase (LCAT) and CETP are key enzymes in HDL metabolism and functionality. LCAT transfers an acyl group from lecithin to free cholesterol, forming cholesterol esters that move to the core of the HDL particle and are eventually delivered to the liver $(12,13)$. Meanwhile, CETP mediates the transfer of cholesterol esters from $\mathrm{HDL}$ to apoB lipoproteins, while triglycerides are simultaneously transferred in the opposite direction (14). Subsequently, the apoB-containing lipoproteins that received cholesterol esters are taken up by the liver, completing the indirect pathway of RCT (15).

The concentration and activity of LCAT and CETP can be modulated by different molecules. For example, polyunsaturated fatty acids (PUFA) reduce transcription of the LCAT mRNA and synthesis of the LCAT protein in vitro (16). On the other hand, sphingomyelin (17), oxidized lipids (18,19), n-3 fatty acids (20) and trans-unsaturated fatty acids (21) inhibit LCAT in vitro. Factors associated with upregulation of CETP gene expression are a high dietary intake of cholesterol of omega-3 PUFA, and the use of fibrates $(22,23)$. Meanwhile, plasma CETP activity and mass are increased upon increases in the plasma concentrations of bile acids (24), and decreased in patients with hypothyroidism (25). Nonetheless, the modulation of human LCAT and CETP by components of HDL in vivo is insufficiently understood.

All lipoproteins harbor a repertoire of small apolipoproteins, which are important modulators of their metabolism. Two of these small apolipoproteins are apoE and apoC-III. ApoE mediates the clearance of VLDL, IDL and chylomicron remnants via the LDL receptor, LDL receptor-related protein-1 (LRP-1) or heparan sulfate proteoglycans $(26,27)$. Contrastingly, apoC-III strongly inhibits the clearance of all apoBlipoproteins $(28,29)$. ApoC-III also impairs the catabolism of triglyceride-rich lipoproteins and stimulates hepatic VLDL assembly and secretion $(28,30)$. However, the role of apoE and apoC-III on HDL physiology is less understood. It is known that besides apoA-I (the natural cofactor of LCAT) (31), apoE is able to activate LCAT in vitro (32), while an increase in the apoC-III content of synthetic HDL shows the opposite effect (33). In apoA-I knockout mice, apoE is able to partially rescue LCAT activity, albeit in VLDL and LDL, not in HDL (34). Despite their major relevance in lipoprotein metabolism, little is known about the influence of apoE and apoC-III over LCAT and CETP in humans.

With this background, we analyzed the distribution of LCAT and CETP mass and activity in multiple HDL subclasses defined by their content of apoE and apoC-III and molecular size, in normal weight and overweight adult humans of both sexes.

\section{Methods}

\section{Study Design and Participants}

We studied 18 patients ( 8 male, 10 female) aged 33 to 76 , selected from a project about new biomarkers of insulin resistance (34). We excluded individuals with known diabetes mellitus or use of anti-diabetic medications, other endocrine disorders, diseases of the exocrine pancreas, pregnant women or patients who received oral anticoagulants or lipid-lowering drugs. Patients who had symptoms of an acute viral or bacterial infection, or in whom hsCRP levels were higher than $10 \mathrm{mg} / \mathrm{L}$, were also excluded. We obtained blood samples in EDTA tubes after an 8 hours fast. Plasma was promptly separated, supplemented with 
a preserving cocktail (benzamidine, phenylmethylsulphonyl fluoride and gentamicin), aliquoted and stored at $-80^{\circ} \mathrm{C}$ for later analyses. We measured plasma concentrations of apoA-I using a nephelometric method (35) and fasting plasma glucose (FPG), plasma lipids and creatinine using conventional colorimetric assays (Biosystems, Spain). Glycated hemoglobin A1c (HbA1c) was determined using a National Glycohemoglobin Standardization Program-certified boronate affinity technique (NycoCard ${ }^{\mathrm{TM}}$ Reader II, Alere Technologies, Norway).

\section{HDL isolation and separation}

Plasma was passed through Acrodisc $® 5 \mu \mathrm{m}$ filters (Pall Corporation, Port Washington, NY), in order to remove fibrin impurities. Then, HDL was purified from plasma using immunoaffinity chromatography as follows: Sepharose $4 \mathrm{~B}^{\mathrm{TM}}$ resin bound to goat polyclonal anti-human apoA-I antibodies (Academy Biomedical $\mathrm{Co}$ ) was loaded into $10 \mathrm{~mL}$ Poly-Prep ${ }^{\circledR}$ chromatography columns (Bio-Rad Laboratories), and 1 $\mathrm{mL}$ of plasma was incubated overnight in the column. The unbound fraction was collected by gravity flow and stored at $-80^{\circ} \mathrm{C}$. The bound fraction was eluted using 3 consecutive washes of $3 \mathrm{M}$ sodium thiocyanate ( $\mathrm{NaSCN})$ and one last PBS wash followed by concentration and desalting in $10 \mathrm{KDa} \mathrm{MW}$ cutoff Amicon ${ }^{\circledR}$ filters (Merck Millipore, Billerica, MA) until $1 \mathrm{~mL}$ of fraction was achieved. Then two PBS washes were performed to remove any NaSCN left over in the column before another use. The same process was carried out with columns containing goat polyclonal anti-human apoC-III and anti-human apo-E antibodies, in that order. At the end, HDL from each plasma sample was divided into 4 subfractions: HDL without apoE or apoC-III (E-C-), HDL with apoE but without apoC-III (E+C-), HDL without apoE but with apoC-III (E-C+) and HDL with both apoE and apoC-III $(E+C+)$. The efficiency of the immunoaffinity columns was $95-98 \%$ for all study subjects. The final buffer for all HDL subfractions was PBS.

\section{Determination of enzymatic content and activity in HDL subfractions}

LCAT concentration was determined using an immunoenzymatic, double-sandwich assay (ALPCO Diagnostics, Salem, $\mathrm{NH}$ ) in which capture is performed by a first monoclonal antibody against LCAT (MoAb 36486) and detection is performed with a different, horseradish peroxidase (HRP)-labeled antiLCAT monoclonal antibody (MoAb 36487). After incubation with a substrate solution and termination with a stop reagent, the intensity of absorbance at $492 \mathrm{~nm}$ was read in a Synergy HT microplate reader using Gen5 software (BioTek, Winooski, VT). CETP concentration was determined using an immunoenzymatic sandwich assay (ALPCO Diagnostics, Salem, NH), with MoAb 3-11D as capture antibody and HRP-labeled MoAb 14-8F as detection antibody.

Measurement of LCAT activity was done using a fluorometric assay (Calbiochem, Darmstadt, Germany). The assay is based on the incubation of a substrate that fluoresces at $470 \mathrm{~nm}$ with the study samples. 
After LCAT in the samples removes a fatty acid from the substrate and transfers it to cholesterol, the substrate loses fluorescence at $470 \mathrm{~nm}$ and gains fluorescence at $390 \mathrm{~nm}$. Thus, LCAT activity was measured as change in 470/390 emission intensity by comparison against a calibration curve based on dilutions of a plasma pool. CETP was also measured with a fluorometric assay (Abcam, Cambridge, UK). The assay principle involves incubating the sample with a mixture that contains a self-quenched fluorescent neutral lipid and an acceptor molecule. Action of CETP in the sample results in transfer of the neutral lipid to the acceptor molecule and an increase in its fluorescence (excitation at $465 \mathrm{~nm}$, emission at $535 \mathrm{~nm}$ ). For CETP too, activity was measured by comparison against a calibration curve based on dilutions of a plasma pool. Enzymatic activity was expressed as percent of activity in the plasma pool, and was therefore expressed in Arbitrary Units (AU). All measurements were performed in duplicate.

The cholesteryl ester content of plasma and HDL subfractions was measured using the Abcam cholesteryl ester kit (ab65359). The assay is based on two simultaneous reactions for cholesterol determination, one of which includes cholesterol esterase as part of the reaction mixture, while the other does not. Cholesteryl esters are then calculated by subtraction as (total cholesterol - free cholesterol). Phosphatidylcholine in plasma and HDL subfractions was measured using the Abcam fluorometric assay (ab83377). The test is based on an enzyme-coupled reaction that hydrolyzes phosphatidylcholine and releases choline, which in turn reacts with the OxiRed probe and generates fluorescence at $587 \mathrm{~nm}$ wavelength.

\section{Determination of HDL size distribution}

HDL contained in each of the four apoC-III and apoE-defined immunofractions were separated by size using non-denaturing polyacrylamide gradient gel electrophoresis (NDPAGGE). Twenty-five microliters of each immunofraction plus 25 microliters of sample buffer were loaded into a 4-30\% polyacrylamide gradient gel (Jule Inc., Milford, CT). Wells 1 and 10 were loaded with molecular size standards (Amersham HMW Native Marker Kit, GE Healthcare, Little Chalfont, UK) and gels were run for 24 hours at constant 70V. Then, contents of the gel were transferred to a 0.45 micrometer pore size polyvinylidene fluoride (PVDF) membrane (Pall Corporation) in a wet transfer apparatus at $30 \mathrm{~V}$ for 24 hours. The lanes containing the $\mathrm{MW}$ markers were cut from the rest of the membrane, stained in $0.2 \%$ amido black solution for 20 minutes and stored for later photographing. The rest of the membrane was blocked with $5 \%$ powder low-fat milk, incubated with an HRP-conjugated goat anti-human apoA-I antibody (Academy Biomedical, Houston, TX), and revealed using 3,3',5,5'-tetramethylbenzidine as substrate. Later, the marker lanes and the rest of the membrane were placed side to side and photographed in a Bio-Rad ChemiDoc ${ }^{\mathrm{TM}}$ MP gel documenter. Using the molecular size standards as reference, the intensity of the bands/smears in each size range fractions was quantitated in Image $\mathrm{Lab}^{\mathrm{TM}}$ software. Size fractions were defined as follows: prebeta HDL: $<7.1 \mathrm{~nm}$, alpha 3 HDL: 7.1-8.2 nm, alpha 2 HDL: 8.2-9.5 nm and alpha $1 \mathrm{HDL}$ : 9.5$12.2 \mathrm{~nm}$. The concentration of apoA-I in each HDL size subfraction was estimated by multiplying the proportion of apoA-I within that fraction by the directly measured total plasma apoA-I. Laboratory 
procedures were executed at the Diabetes, Lipids and Metabolism laboratory of Universidad de Los Andes, following current institutional biosafety protocols.

\section{Statistical analyses}

Our sample size of eighteen participants gave us $87 \%$ power to detect a true difference of at least $8 \%$ in LCAT activity between two HDL subtypes, assuming a $5 \%$ variation coefficient in LCAT activity (18), at a $5 \%$ significance level. The distribution of plasma apoA-I across HDL subfractions was compared in a 2way ANOVA model in which apoA-I concentration in each subfraction was the dependent variable, and HDL size and immunofraction were fixed factors. Enzyme masses and activity across HDL subtypes were compared using a 1-way ANOVA in which HDL immunofraction was the only fixed factor. When global ANOVA was significant, post-hoc comparisons against the reference fraction (E-C-) were done using Scheffé's method. Comparisons of quantitative variables between groups were performed using MannWhitney's U test. Differences in categorical variables between groups were performed using Fisher's exact test. All tests were done at a 0.05 significance level and all reported p-values are 2 -sided.

\section{Results}

The study included 8 male and 10 female adults, with mean age $55.6+/-11.2$ years and mean bodymass index (BMI) $26.9+/-4.0 \mathrm{~kg} / \mathrm{m}^{2}$. Women had higher plasma total cholesterol, HDLc and LDLc. Women also had higher plasma apoA-l levels $(121.4+/-14.3 \mathrm{mg} / \mathrm{dL}$ versus $97.7+/-23.0 \mathrm{mg} / \mathrm{dL}$ in men, $\mathrm{p}=0.016$ ). Total plasma LCAT was $6.3+/-2.2 \mathrm{mg} / \mathrm{dL}$ in women and $5.7+/-2.2 \mathrm{mg} / \mathrm{dL}$ in men, while plasma CETP concentrations were $1.9+/-0.7 \mathrm{mg} / \mathrm{dL}$ in women and $1.7+/-0.7 \mathrm{mg} / \mathrm{dL}$ in men. Relative plasma CETP activity was very similar in both sexes, 98.7 +/- $13.7 \mathrm{AU}$ in women and 101.7 +/- $6.1 \mathrm{AU}$ in men. Meanwhile, LCAT activity was $96.1+/-22.3 \mathrm{AU}$ in women and $96.8+/-18.4$ AU in men (Table 1).

\section{HDL with apoE or apoC-III are minority}

The predominant subfraction of HDL (as reflected by its concentration of apoA-I) was E-C- in most study participants. On average, apoA-I in E-C- HDL was the most abundant HDL subtype, representing $50.1 \%$ of plasma apoA-I, followed by E+C- (22.6\%), E-C+ (15.2\%) and E+C+ (12.1\%) ( $<<0.001$ for difference across subfractions) (Figure 1). There was significantly more apoA-I in E-C- than in E+C-, E-C+ or E+C+ HDL ( $p<0.001$ for each of the three pairwise comparisons). Overall, $34.7 \%$ of HDL contained any apoE while $27.3 \%$ contained any apoC-III. 
Most plasma LCAT mass (3.58 microg/mL, 59.6\%) was concentrated in the E-C- HDL subfraction. The remaining fractions with either apoE or apoC-III had similarly lower LCAT concentrations: $1.19 \mathrm{microg} / \mathrm{mL}$ in $\mathrm{E}+\mathrm{C}-, 0.74 \mathrm{microg} / \mathrm{mL}$ in $\mathrm{E}-\mathrm{C}+$ and $0.50 \mathrm{microg} / \mathrm{mL}$ in $\mathrm{E}+\mathrm{C}+$ ( $p<0.001$ for the difference across groups). The difference in LCAT concentration between E-C- and every other HDL subfraction was statistically significant ( $p<0.001$ for each of the three comparisons) (Figure 2, upper panel). Surprisingly, this heterogeneity in LCAT protein distribution was not paralleled by LCAT activity, which had a different distribution across HDL subfractions. The E-C- fraction had a relative LCAT activity of $24.1 \mathrm{AU}$ ( $25 \%$ of total plasma LCAT activity), while the highest LCAT activity was found in the in E+C- fraction (26.5 AU, $27.4 \%$ of total plasma LCAT activity) ( $p=0.35$ for difference across groups). HDL in the $\mathrm{E}-\mathrm{C}+$ and $\mathrm{E}+\mathrm{C}+$ fractions contained respectively 25.8 and 20.0 AU of LCAT activity (Figure 2, lower panel). The ratio of LCAT activity / LCAT mass was $27.5 \mathrm{AU} * \mathrm{~mL} /$ microg in $\mathrm{HDL}$ containing apoE ( $\mathrm{E}+\mathrm{C}$ - plus $\mathrm{E}+\mathrm{C}+$ ), and 11.6 $\mathrm{AU} * \mathrm{~mL} /$ microg for HDL not containing apoE (E-C-plus E-C+).

CETP concentration and activity are similar across HDL subfractions

Most CETP was concentrated in the $\mathrm{E}+\mathrm{C}$ - subfraction $(0.60 \mathrm{mg} / \mathrm{mL}, 32.5 \%$ of total plasma CETP), while the other three types of HDL contained respectively $25.9 \%$ (E-C-), 21.1\% (E-C+) and $20.4 \%(E+C+)$ of plasma CETP ( $p=0.49$ for comparison across fractions) (Figure 3 , upper panel). CETP activity showed a very homogeneous distribution across HDL subtypes ( $p=0.50$, Figure 3 , lower panel).

\section{Lipid contents of HDL subtypes}

ApoC-III containing HDL displayed a higher triglyceride-to-apoA1 molar ratio, while E-C- HDL were poorer in triglycerides relative to the other fractions ( $p=0.01$ for global ANOVA, Table 2$)$. Despite numerically higher phosphatidylcholine-to-apoA1 molar ratios in the apoE or apoC-III containing HDL, this difference did not achieve statistical significance. The cholesterol and cholesteryl ester contents of the four HDL subtypes were numerically similar, and not statistically different.

The concentration of some apolipoprotein and size-defined HDL correlates with plasma LCAT and CETP activity

The concentration of apoA-I in the E-C+ pre-beta HDL subfraction correlated inversely with total plasma LCAT activity ( $r=-0.55, p=0.017$, Table 3$)$. Similarly, apoA-I in the E-C- alpha $1 \mathrm{HDL}$ subfraction correlated inversely with total plasma LCAT activity $(r=-0.49, p=0.041)$. On the other hand, larger $E-C+H D L$ (alpha 1) exhibited a positive correlation with plasma CETP activity $(r=0.52, p=0.025)$. For the E-C+ HDL subtype, the correlation between HDL concentration and CETP activity was not significant for smaller HDL, but tended to increase along with HDL size. 
As a sensitivity analysis, we examined whether the findings concerning enzymatic concentration and activities were different for participants with a normal body-mass index (BMIX25), compared to those with excess body weight (overweight or obesity, $B M I \nabla=25$ ). Just like in the compete study sample, in each of the two groups the concentration of LCAT was significantly higher in E-C- HDL than in the other fractions, but enzymatic activity was similarly distributed across HDL subtypes (Figure 4). Also in each of the two BMI-defined subgroups, CETP concentration and activity were very similar across the four HDL subtypes.

\section{Discussion}

In this study of human HDL, we found that HDL subtypes defined by their apoE and apoC-III content have different LCAT mass but comparable LCAT activity. In other words, we encountered that HDL with apoE have a greater ratio of LCAT activity to mass, signaling a greater degree of LCAT activation. Contrastingly, CETP mass and activity were not associated with the apoE or apoC-III composition of HDL. As reported previously, apoE and/or apoC-III-containing HDL were a minority of total plasma HDL (36), yet they proved to be different from E-C- HDL in their enzymatic activity.

LCAT was most abundant in E-C- HDL, while HDL with either apoE or apoC-III contained similar amounts of LCAT. Despite having lower LCAT masses compared to E-C- HDL, subfractions with apoE or apoC-III had very similar LCAT activities, suggesting that the presence of either small apolipoprotein, or some factor closely associated with them, influences LCAT. Of note, the simultaneous presence of apoE and apoC-III was not accompanied by a higher LCAT activity. Unlike apoA-I (the essential cofactor of LCAT), very little is known about the impact of other apolipoproteins of the small, exchangeable type on the activity of this crucial HDL enzyme. Studies in apoA-l-/- apoE-/- double knockout mice have identified apoE as a potential LCAT activator (37). ApoE is also able to activate LCAT in discoidal reconstituted HDL devoid of apoA-I (38). In contrast, apoC-III dose-dependently inhibits the LCAT reaction in reconstituted HDL (33), and in synthetic phosphatidylcholine vesicles containing apoA-I (39). Therefore, the finding of no decrease in LCAT activity in apoC-III containing HDL was unexpected.

Contrary to LCAT, CETP concentration and activity did not vary among HDL subfractions. Although some CETP inhibitors have the ability to increase both plasma apoC-III and plasma apoE (40-42), our findings suggest that CETP activity and concentration in HDL are not influenced by these apolipoproteins. CETP activity in humans may be influenced by high plasma cholesterol concentrations, or by cholestasis $(43,44)$. 
Concerning the lipid composition of HDL subfractions, we found more phosphatidylcholine in HDL with either apoE or apoC-III. Thus, higher LCAT activity in these HDL subtypes may be due to greater availability of substrate. This explanation has biological plausibility, as the movement of apolipoproteins between lipoproteins sometimes involves phospholipid transfer protein (PLTP), in which cases there may be simultaneous transfer of the surrounding phospholipid-rich membrane (45). We did not find statistically significant differences in cholesteryl ester content among the four HDL subtypes.

We found a significant inverse correlation between total plasma LCAT activity and the concentration of both E-C+ pre-beta HDL and E-C- alpha $1 \mathrm{HDL}$. These results suggests that the presence of apoC-III in prebeta HDL may correlate with limited LCAT activity, less maturation and accumulation of pre-beta HDL (46). Interestingly, a study comparing patients with and without CVD found an inverse correlation between LCAT activity and plasma pre-beta HDL concentrations, which was stronger in the CVD group (47). Our finding of a negative correlation between E-C- alpha $1 \mathrm{HDL}$ and plasma LCAT activity suggests that not all size conversion/maturation of HDL requires LCAT. Meanwhile, the positive correlation of plasma CETP activity with the abundance of large E-C+ HDL may just reflect the frequent coexistence of both proteins (CETP and apoC-III) in this HDL type.

The main limitations of our study are its limited sample size and the fact that we interrogated only two specific apolipoproteins, out of the many known to be present in HDL. However, this was a proof-ofconcept study in which the core analyses were performed within-individual, so that enzymatic contents and activities were performed for different HDL subtypes belonging to the same individual. The biological antagonism between apoE and apoC-III (48) has also been proven to modulate HDL metabolism and its association with cardiovascular risk (49), making them particularly worthy of investigation, particularly because the influence of apoE on HDL metabolism has been proven to be susceptible of positive modification through dietary interventions (50). Another possible limitation is that the employed LCAT activity assay probably detects only the fatty acid removal from PC and not its successful transfer to free cholesterol. Nonetheless, given the known nature of the LCAT enzymatic process, the ability of LCAT to perform this initial cleavage in vitro has been widely employed as a proxy for LCAT activity.

In conclusion, our results showed specific profiles of LCAT mass and activity in HDL subtypes defined by their content of apoE and apoC-III, and suggest that the presence of apoE protein in HDL may be a correlate of increased LCAT activity. Further studies describing the changes in HDL composition, functionality and associated enzymatic activity in the context of CVD would expand on our findings.

\section{List Of Abbreviations}


apoC-III: apolipoprotein C-III

apoE: apolipoproteins E

AU: Arbitrary Units

BMI: body-mass index

CETP: cholesterol-ester transfer protein

CVD: cardiovascular disease

HDL: High density lipoproteins

HRP: horseradish peroxidase

LCAT: lecithin-cholesterol acyl transferase

LRP-1: LDL receptor-related protein-1

RCT: reverse cholesterol transport

PUFA: Polyunsaturated fatty acids

PVDF: polyvinylidene fluoride

\section{Declarations}

\section{Ethics approval and consent to participate}

The Internal Review Board (Comité de Ética) of Universidad de los Andes approved the study according to minute 307 of 2014. We complied with all scientific, technical and administrative norms for health research dictated by resolution 008430 - 1993 of the Colombian Ministry of Health and with the principles stated by the Declaration of Helsinki. All study subjects underwent an informed consent procedure and provided written informed consent.

Consent for publication

Not applicable 
The datasets used and/or analysed during the current study are available from the corresponding author on reasonable request.

\section{Competing interests}

The authors declare that they have no competing interests

\section{Funding}

This study was funded by Vicerrectoría de Investigaciones, Universidad de los Andes, Colombia

\section{Authors' contributions}

MA-M, JAP-C, LSS-B and COM participated in the study conception, MA-M, JAP-C, LSS-B, DR-M, MCPM, MAJM and COM executed study experiments and data collection, MA-M, DR-M and COM participated in data analysis and manuscript writing, COM directed the project and participated in all phases of its conception, execution, and in the manuscript writing.

\section{Acknowledgements}

We want to thank the study participants for their patience and collaboration.

\section{References}

1. Rosenson RS, Brewer HB Jr, Barter PJ, Björkegren JLM, Chapman MJ, Gaudet D, Kim DS, Niesor E, Rye KA, Sacks FM, Tardif JC, Hegele RA. HDL and atherosclerotic cardiovascular disease: genetic insights into complex biology. Nat Rev Cardiol. 2018;15:9-19.

2. Vitali C, Khetarpal SA, Rader DJ. HDL cholesterol metabolism and the risk of CHD: New insights from human genetics. Curr Cardiol Rep. 2017;19:132.

3. Nicholls SJ, Nelson AJ. HDL and cardiovascular disease. Pathology 2019;51:142-147.

4. Shahid M, Sun RL, Liu Y, Bao JL, Huang CX, Liao Y, Zhou SX, Wang JF, Zhang YL. Is high high-density lipoprotein cholesterol beneficial for premature coronary heart disease? A meta-analysis. Eur J Prev Cardiol. 2016;23:704-713.

5. Barter P, Caulfield M, Eriksson M, Grundy S, Kastelein J, Komajda M, Lopez-Sendon, J, Mosca L, Tardif J, Waters D, Shear C, Revkin J, Buhr K, Fisher M, Tall A and Brewer B. Effects of Torcetrapib in 
Patients at High Risk for Coronary Events. N Engl J Med. 2007;357:2109-2122.

6. Schwartz G, Olsson A, Abt M, Ballantyne C, Barter P, Brumm J et al. Effects of Dalcetrapib in patients with a recent acute coronary syndrome. N Engl J Med. 2012;367:2089-2099.

7. Landray M, Haynes R, Hopewell J, Parish S, Phil D, Aung T et al. Effects of extended-release niacin with laropiprant in high-risk patients. N Engl J Med. 2014;371:203-212.

8. Lincoff A, Nicholls S, Riesmeyer J, Barter P, Brewer H, Fox K et al. Evacetrapib and cardiovascular outcomes in high-risk vascular disease. N Engl J Med. 2017;376:1933-1942.

9. Bardagjy AS, Steinberg FM. Relationship between hdl functional characteristics and cardiovascular health and potential impact of dietary patterns: A narrative review. Nutrients 2019;11:E1231.

10. Cuchel M, Rohatgi A, Sacks FM, Guyton JR. JCL roundtable: High-density lipoprotein function and reverse cholesterol transport. J Clin Lipidol 2018;12:1086-1094.

11. Rosenson R, Brewer H, Ansell B, Barter P, Chapman M, Heinecke J et al. Dysfunctional HDL and atherosclerotic cardiovascular disease. Nat Rev Cardiol. 2016;13:48-60.

12. Dobiášová $M$. Atherogenic impact of lecithin-cholesterol acyltransferase and its relation to cholesterol esterification rate in HDL (FER(HDL)) and AIP [log(TG/HDL-C)] biomarkers: the butterfly effect? Physiol Res. 2017;66:193-203.

13. Mendivil CO, Furtado J, Morton AM, Wang L, Sacks FM. Novel pathways of apolipoprotein A-I metabolism in high-density lipoprotein of different sizes in humans. Arterioscler Thromb Vasc Biol. 2016;36:156-165.

14. Barter P, Rye K. Cholesteryl Ester Transfer Protein inhibition is not yet dead-pro. Arterioscler Thromb Vasc Biol. 2016;36:439-441.

15. Maranhão RC, Freitas FR. HDL metabolism and atheroprotection: predictive value of lipid transfers. Adv Clin Chem 2014;65:1-41.

16. Kuang YL, Paulson KE, Lichtenstein AH, Lamon-Fava S. Regulation of the expression of key genes involved in HDL metabolism by unsaturated fatty acids. Br J Nutr. 2012;108:1351-1359.

17. Subbaiah PV, Jiang XC, Belikova NA, Aizezi B, Huang ZH, Reardon CA. Regulation of plasma cholesterol esterification by sphingomyelin: effect of physiological variations of plasma sphingomyelin on lecithin-cholesterol acyltransferase activity. Biochim Biophys Acta. 2012;1821:908913.

18. Nakhjavani M, Asgharani F, Khalilzadeh O, Esteghamati A, Ghaneei A, Morteza A, Anvari M. Oxidized low-density lipoprotein is negatively correlated with lecithin-cholesterol acyltransferase activity in type 2 diabetes mellitus. Am J Med Sci. 2011;341:92-5.

19. Bielicki JK, Forte TM. Evidence that lipid hydroperoxides inhibit plasma lecithin:cholesterol acyltransferase activity. J Lipid Res. 1999;40:948-954.

20. Abbey M, Clifton P, Kestin M, Belling B, Nestel P. Effect of fish oil on lipoproteins, lecithin: cholesterol acyltransferase, and lipid transfer protein activity in humans. Arteriosclerosis. 1990;10:85-94. 
21. Subbaiah PV, Subramanian VS, Liu M. Trans unsaturated fatty acids inhibit lecithin: cholesterol acyltransferase and alter its positional specificity. J Lipid Res. 1998;39:1438-1447.

22. Jiang XC, Agellon LB, Walsh A, Breslow JL, Tall A. Dietary cholesterol increases transcription of the human cholesteryl ester transfer protein gene in transgenic mice. Dependence on natural flanking sequences. J Clin Invest. 1992;90:1290-1295.

23. Raposo HF, Patrício PR, Simões MC, Oliveira HC. Fibrates and fish oil, but not corn oil, up-regulate the expression of the cholesteryl ester transfer protein (CETP) gene. J Nutr Biochem. 2014;25:669-674.

24. Gautier T, de Haan W, Grober J, Ye D, Bahr MJ, Claudel T, et al. Farnesoid X receptor activation increases cholesteryl ester transfer protein expression in humans and transgenic mice. J Lipid Res. 2013;54:2195-2205.

25. Skoczyńska A, Wojakowska A, Turczyn B, Zatońska K, Wołyniec M, Rogala N, Szuba A, BednarekTupikowska G. Serum Lipid Transfer Proteins in Hypothyreotic Patients Are Inversely Correlated with Thyroid-Stimulating Hormone (TSH) Levels. Med Sci Monit. 2016;22:4661-4669.

26. Huang Y, Mahley RW. Apolipoprotein E: Structure and function in lipid metabolism, neurobiology, and Alzheimer's diseases. Neurobiol Dis. 2014;72:3-12.

27. Mondal T, Wang H, DeKoster GT, Baban B, Gross ML, Frieden C. ApoE: In vitro studies of a small molecule effector. Biochemistry. 2016;55:2613-21.

28. Zheng C, Khoo C, Furtado J, Sacks FM. Apolipoprotein C-III and the metabolic basis for hypertriglyceridemia and the dense low-density lipoprotein phenotype. Circulation. 2010;121:17221734.

29. Mendivil CO, Zheng C, Furtado J, Lel J, Sacks FM. Metabolism of very-low-density lipoprotein and low-density lipoprotein containing apolipoprotein C-III and not other small apolipoproteins. Arterioscler Thromb Vasc Biol. 2010;30:239-245.

30. Qin W, Sundaram M, Wang Y, Zhou H, Zhong S, Chang CC, Manhas S, Yao EF, Parks RJ, McFie PJ, Stone SJ, Jiang ZG, Wang C, Figeys D, Jia W, Yao Z. Missense mutation in APOC3 within the Cterminal lipid binding domain of human ApoC-III results in impaired assembly and secretion of triacylglycerol-rich very low density lipoproteins: evidence that ApoC-III plays a major role in the formation of lipid precursors within the microsomal lumen. J Biol Chem. 2011;286:27769-27780.

31. Sorci-Thomas MG, Bhat S, Thomas MJ. Activation of lecithin:cholesterol acyltransferase by HDL ApoA-I central helices. Clin Lipidol. 2009;4:113-124.

32. Jonas A. Lecithin cholesterol acyltransferase. Biochim Biophys Acta. 2000;1529:245-256.

33. Cho KH. Synthesis of reconstituted high density lipoprotein ( $\mathrm{rHDL}$ ) containing apoA-I and apoC-III: the functional role of apoC-III in rHDL. Mol Cells. 2009;27:291-297.

34. Toloza FJK, Pérez-Matos MC, Ricardo-Silgado ML, Morales-Álvarez MC, Mantilla-Rivas JO, PinzónCortés JA, Pérez-Mayorga M, Arévalo-García ML, Tolosa-González G, Mendivil CO. Comparison of plasma pigment epithelium-derived factor (PEDF), retinol binding protein 4 (RBP-4), chitinase-3-like 
protein 1 (YKL-40) and brain-derived neurotrophic factor (BDNF) for the identification of insulin resistance. J Diabetes Complications. 2017;31:1423-1429.

35. Hanson NQ, Freier EF. Nephelometric assay of apolipoprotein A-I with a centrifugal analyzer. Clin Chem. 1986;32:2090-2093.

36. Talayero B, Wang L, Furtado J, Carey VJ, Bray GA, Sacks FM. Obesity favors apolipoprotein E- and CIII-containing high density lipoprotein subfractions associated with risk of heart disease. J Lipid Res. 2014;55:2167-2177.

37. Zhao Y, Thorngate FE, Weisgraber KH, Williams DL, Parks JS. Apolipoprotein E is the major physiological activator of lecithin-cholesterol acyltransferase (LCAT) on apolipoprotein B lipoproteins. Biochemistry. 2005;44:1013-1025.

38. Rye KA, Bright R, Psaltis M, Barter PJ. Regulation of reconstituted high density lipoprotein structure and remodeling by apolipoprotein E. J Lipid Res. 2006;47:1025-1036.

39. Nishida HI, Nakanishi T, Yen EA, Arai H, Yen FT, Nishida T. Nature of the enhancement of lecithincholesterol acyltransferase reaction by various apolipoproteins. J Biol Chem. 1986;261:1202812035.

40. Millar JS, Lassman ME, Thomas T, Ramakrishnan R, Jumes P, et al. Effects of CETP inhibition with anacetrapib on metabolism of VLDL-TG and plasma apolipoproteins C-II, C-III, and E. J Lipid Res. 2017; 58:1214-1220.

41. Bagdade J, Barter P, Quiroga C, Alaupovic P. Effects of torcetrapib and statin treatment on apoC-III and apoprotein-defined lipoprotein subclasses (from the ILLUMINATE Trial). Am J Cardiol. 2017;119:1753-1756.

42. Nicholls SJ, Ray KK, Ballantyne CM, Beacham LA, Miller DL, Ruotolo G, et al. Comparative effects of cholesteryl ester transfer protein inhibition, statin or ezetimibe on lipid factors: The ACCENTUATE trial. Atherosclerosis. 2017;261:12-18.

43. Sigal GA, Tavoni TM, Silva BMO, Kalil Filho R, Brandão LG, Maranhão RC. Effects of short-term hypothyroidism on the lipid transfer to high-density lipoprotein and other parameters related to lipoprotein metabolism in patients submitted to thyroidectomy for thyroid cancer. Thyroid. 2019;29:53-58.

44. Iglesias A, Arranz M, Alvarez JJ, Perales J, Villar J, Herrera E, Lasunción MA. Cholesteryl ester transfer activity in liver disease and cholestasis, and its relation with fatty acid composition of lipoprotein lipids. Clin Chim Acta. 1996;248:157-174.

45. Huuskonen J1, Olkkonen VM, Ehnholm C, Metso J, Julkunen I, Jauhiainen M. Phospholipid transfer is a prerequisite for PLTP-mediated HDL conversion. Biochemistry. 2000;39:16092-8.

46. Rousset X, Shamburek R, Vaisman B, Amar M, Remaley A. Lecithin Cholesterol Acyltransferase: An anti- or pro-atherogenic factor? Curr Atheroscler Rep. 2011;13:249-256.

47. Sethi A, Sampson M, Warnick R, Muniz N, Vaisman B, Nordestgaard B et al. High Pre- $\beta 1$ HDL concentrations and low lecithin: cholesterol acyltransferase activities are strong positive risk markers for ischemic heart disease and independent of HDL-cholesterol. Clin Chem. 2010;56:1128-1137. 
48. Sacks FM. The crucial roles of apolipoproteins $E$ and C-III in apoB lipoprotein metabolism in normolipidemia and hypertriglyceridemia. Curr Opin Lipidol. 2015;26:56-63.

49. Morton AM, Koch M, Mendivil CO, Furtado J, Tjønneland A, Overvad K, Wang L, Jensen MK, Sacks FM. Apolipoproteins $\mathrm{E}$ and $\mathrm{CIII}$ interact to regulate HDL metabolism and coronary heart disease risk. JCl Insight. 2018;3:e98045.

50. Morton AM, Furtado JD, Mendivil CO, Sacks FM. Dietary unsaturated fat increases HDL metabolic pathways involving apoE favorable to reverse cholesterol transport. JCI Insight. 2019;4: 124620.

\section{Tables}

Table 1. Characteristics of study participants. eGFR: Estimated glomerular filtration rate. Data are mean +/- SD unless stated otherwise.

\begin{tabular}{|l|c|}
\hline Sex (F:M) & $10: 8$ \\
\hline Age (years) & $55.6+/-11.2$ \\
\hline Weight $(\mathrm{kg})$ & $72.0+/-12.9$ \\
\hline Height $(\mathrm{m})$ & $163.5+/-9.2$ \\
\hline Body-mass index $\left(\mathrm{kg} / \mathrm{m}^{2}\right)$ & $26.9+/-4.0$ \\
\hline Percent body fat $(\%)$ & $33.1+/-7.1$ \\
\hline Percent abdominal fat (\%) & $9.4+/-3.9$ \\
\hline Percent lean mass (\%) & $63.6+/-6.7$ \\
\hline Systolic blood pressure $(\mathrm{mmHg})$ & $117.8+/-14.6$ \\
\hline Diastolic blood pressure (mmHg) & $73.8+/-11$ \\
\hline Fasting plasma glucose (mg/dl) & $96.1+/-10.6$ \\
\hline Glycated hemoglobin (HbA1c) $(\%)$ & $5.4+/-1.0$ \\
\hline eGFR (ml/min) & $93.2+/-18.0$ \\
\hline Total cholesterol (mg/dl) & $195.5+/-52.4$ \\
\hline Triglycerides (mg/dl) & $154.2+/-58.4$ \\
\hline HDL cholesterol (mg/dl) & $43.8+/-15.8$ \\
\hline LDL cholesterol (mg/dl) & $126.3+/-44.4$ \\
\hline C-reactive protein (mg/l) & $1.9+/-2.6$ \\
\hline Plasma apoA-I (mg/dl) & $110.9+/-21.8$ \\
\hline Plasma CETP (microg/ml) & $1.8+/-0.7$ \\
\hline Plasma LCAT (microg/ml) & $6.0+/-2.1$ \\
\hline Plasma CETP activity (AU) & $100.0+/-10.8$ \\
\hline Plasma LCAT activity (AU) & $96.4+/-20.1$ \\
\hline
\end{tabular}

Table 2. Molar ratios of lipids to apoA-I in the four HDL subfractions. Data in the first row are mean lipid to apoA-I molar ratios +/- SD. The second row of each analyte shows the significance values from a posthoc pairwise comparison versus the E-CIII- fraction. The third row of each analyte shows the proportion of each lipid in that fraction as a percentage of the total mass of such lipid in HDL. The last row shows the significance value from a global one-way ANOVA for the analysis of lipid to apoA-I molar ratios by subfractions. 


\begin{tabular}{|c|c|c|c|c|c|}
\hline & & E-CIII- & $\mathrm{E}+\mathrm{CIII-}$ & E-CIII+ & $\mathrm{E}+\mathrm{CIII+}$ \\
\hline \multirow[t]{4}{*}{ Triglycerides } & to apoA-I molar ratio & $0.47+/-0.22$ & $1.09+/-1.41$ & $2.73+/-3.48$ & $2.41+/-1.87$ \\
\hline & p-value versus E-CIII- & - & 0.88 & 0.035 & 0.09 \\
\hline & $\%$ of total mass in HDL & $25.2 \%$ & $17.2 \%$ & $29.8 \%$ & $27.7 \%$ \\
\hline & p-value from ANOVA & \multicolumn{4}{|c|}{0.01} \\
\hline \multirow[t]{4}{*}{ Cholesterol } & to apoA-I molar ratio & $26.6+/-18.7$ & $51.5+/-68.4$ & $48.1+/-32$ & $52.6+/-38.1$ \\
\hline & p-value versus E-CIII- & - & 0.43 & 0.55 & 0.39 \\
\hline & $\%$ of total mass in HDL & $40.7 \%$ & $22.5 \%$ & $20.8 \%$ & $15.8 \%$ \\
\hline & p-value from ANOVA & \multicolumn{4}{|c|}{0.26} \\
\hline \multirow[t]{4}{*}{ Phosphatidylcholine } & to apoA-I molar ratio & $109.2+/-69.3$ & $317.6+/-367.7$ & $455.7+/-547.3$ & $488.2+/-669.2$ \\
\hline & p-value versus E-CIII- & - & 0.65 & 0.21 & 0.15 \\
\hline & $\%$ of total mass in HDL & $27.1 \%$ & $27.0 \%$ & $26.0 \%$ & $19.7 \%$ \\
\hline & p-value from ANOVA & \multicolumn{4}{|c|}{0.088} \\
\hline \multirow[t]{4}{*}{ Cholesterol esters } & to apoA-I molar ratio & $14.6+/-16$ & $23+/-29.9$ & $16.3+/-37.9$ & $30+/-103$ \\
\hline & p-value versus E-CIII- & - & 0.98 & 0.99 & 0.89 \\
\hline & $\%$ of total mass in HDL & $48.6 \%$ & $28.8 \%$ & $12.3 \%$ & $10.1 \%$ \\
\hline & p-value from ANOVA & \multicolumn{4}{|c|}{0.86} \\
\hline
\end{tabular}

Table 3. Correlation between ApoA1 within HDL size-defined subfractions and plasma enzymatic activity. Data are Spearman linear correlation coefficients. Asterisks denote correlations significantly different from zero.

\begin{tabular}{|r|c|c|c|c|}
\hline Correlations with LCAT activity & E-CIII- & E+CIII- & E-CIII+ & E+CIII+ \\
\hline Alpha 1 HDL & $-0.49^{\star}$ & -0.30 & -0.35 & -0.10 \\
\hline Alpha 2 HDL & -0.01 & -0.29 & -0.37 & 0.02 \\
\hline Alpha 3 HDL & 0.33 & -0.13 & -0.30 & 0.04 \\
\hline Pre-Beta HDL & 0.24 & -0.08 & $-0.55^{\star}$ & 0.06 \\
\hline Correlations with CETP activity & E-CIII- & E+CIII- & E-CIII+ & E+CIII+ \\
\hline Alpha 1 HDL & 0.16 & -0.10 & $0.52^{\star}$ & 0.46 \\
\hline Alpha 2 HDL & 0.04 & -0.07 & 0.39 & 0.34 \\
\hline Alpha 3 HDL & -0.03 & 0.11 & 0.28 & 0.28 \\
\hline Pre-Beta HDL & 0.11 & -0.01 & 0.13 & 0.05 \\
\hline
\end{tabular}

\section{Figures}




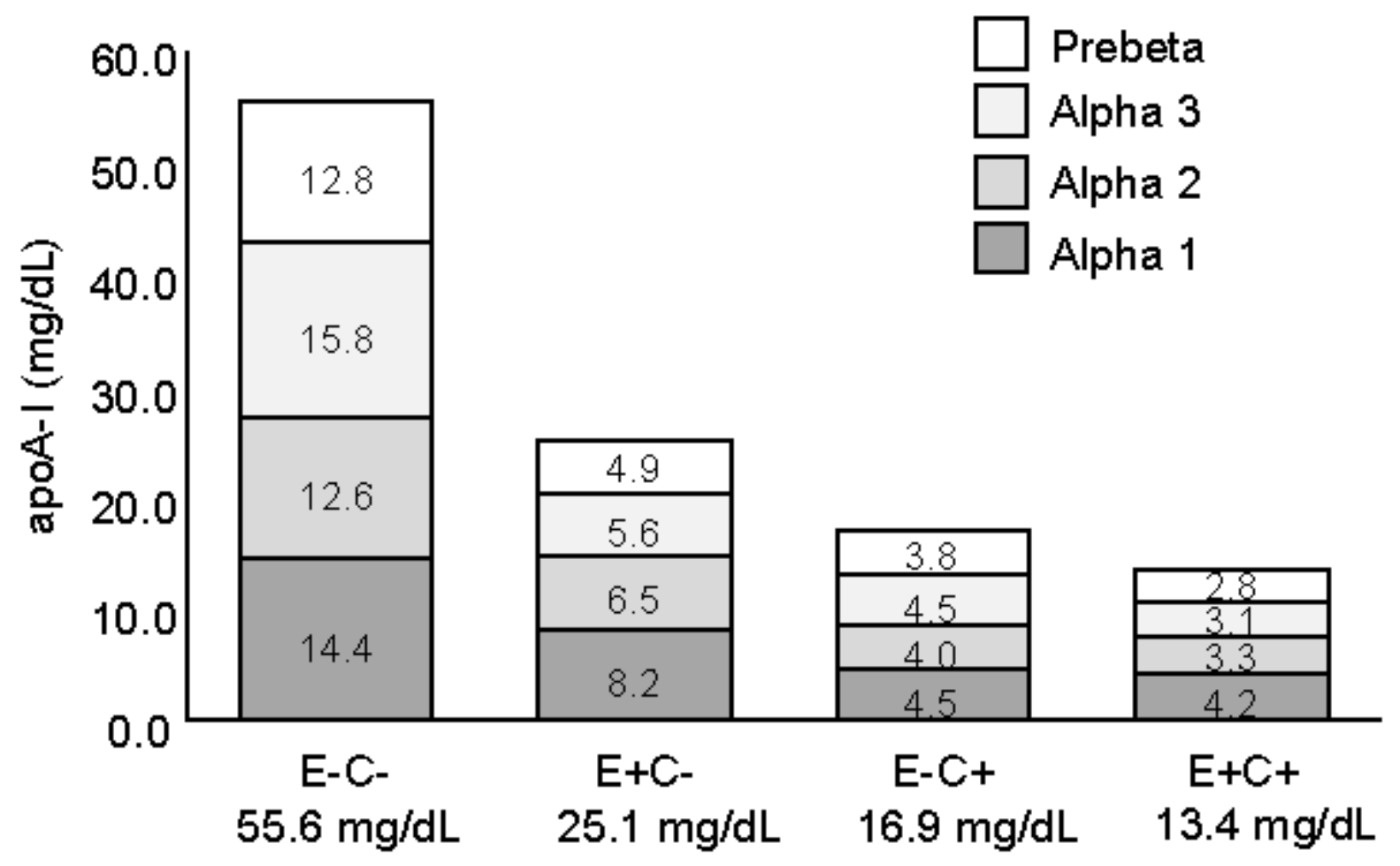

Figure 1

Distribution of plasma apoA-I across HDL subfractions defined by size, apoE and apoC-III content. HDL size ranges are as follows: Prebeta: $<7.1 \mathrm{~nm}$, alpha 3: 7.1-8.2 nm, alpha 2: 8.2-9.5 nm, alpha 1: 9.5-12.2 $\mathrm{nm}(\mathrm{n}=18,10$ women and 8 men). 

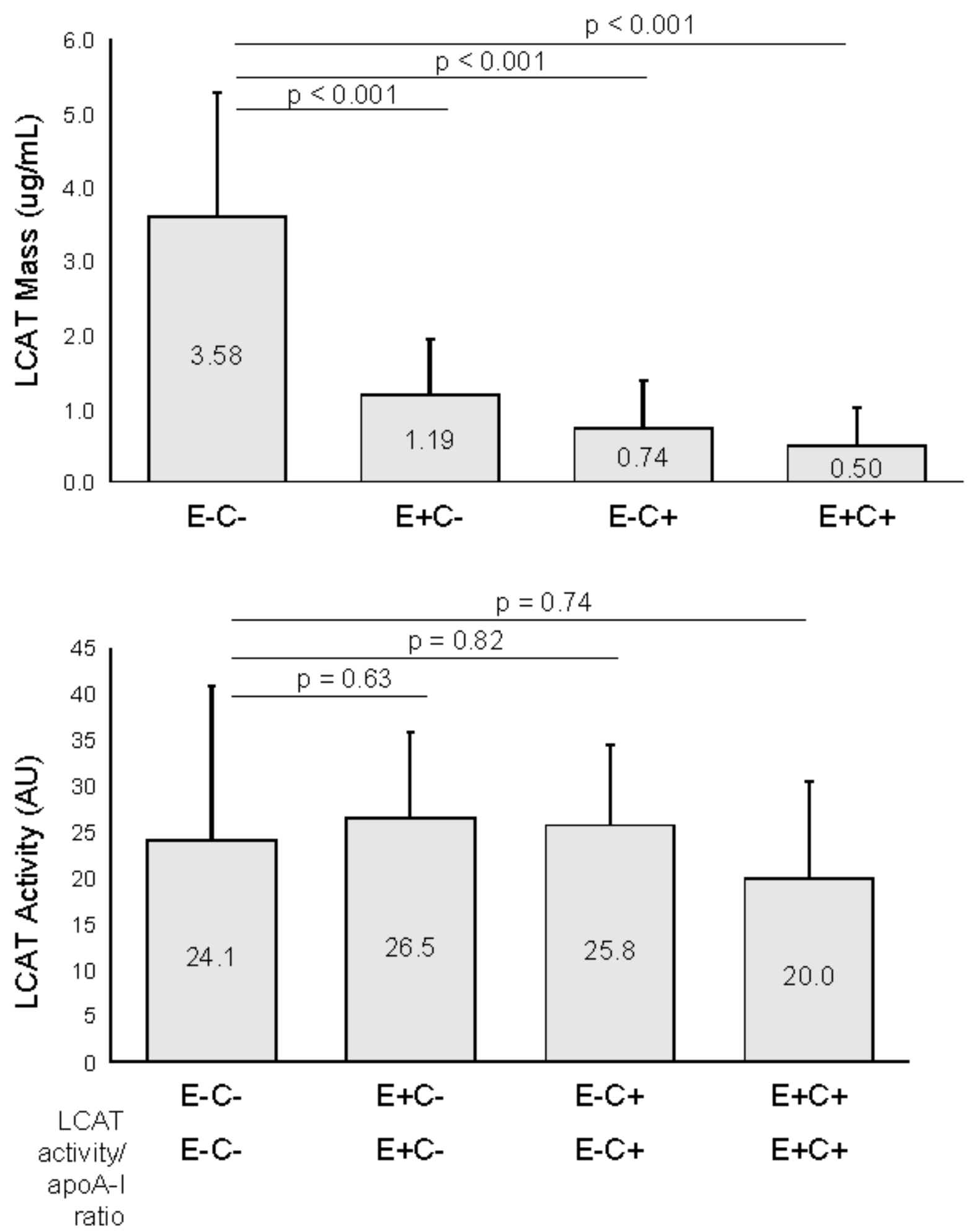

Figure 2

LCAT concentrations and activities in HDL subfractions defined by their apoE and apoC-III content. (ATop) LCAT concentrations. (B-bottom) LCAT activities ( $n=18,10$ women and 8 men). 

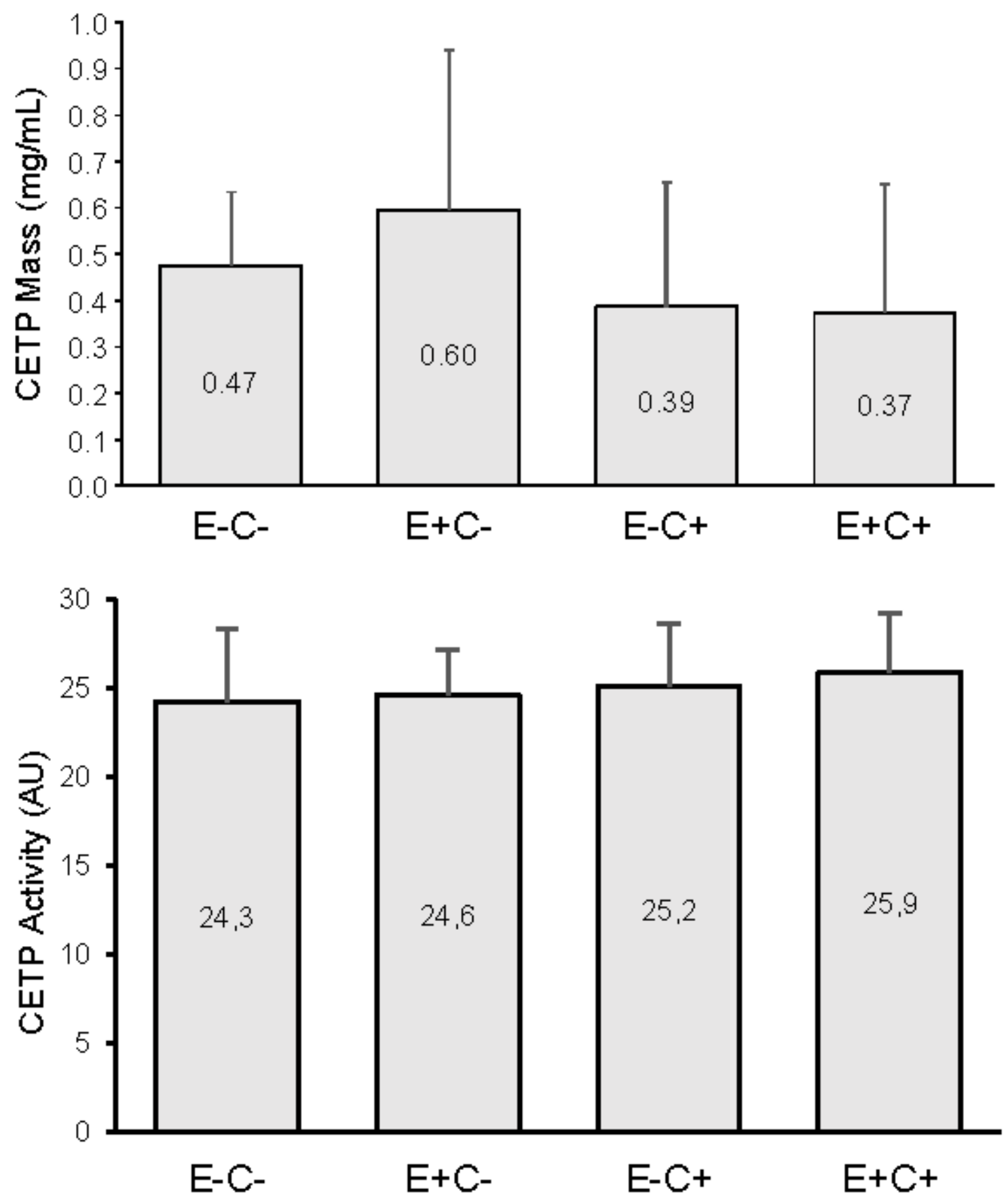

Figure 3

CETP concentrations and activities in HDL subfractions defined by their apoE and apoC-III content. (Atop) CETP concentrations. There was no significant difference across subfractions (overall ANOVA $p=0.48$ ). (B-bottom) CETP activities. There was no significant difference across subfractions (overall ANOVA $p=0.50)(n=18,10$ women and 8 men $)$. 


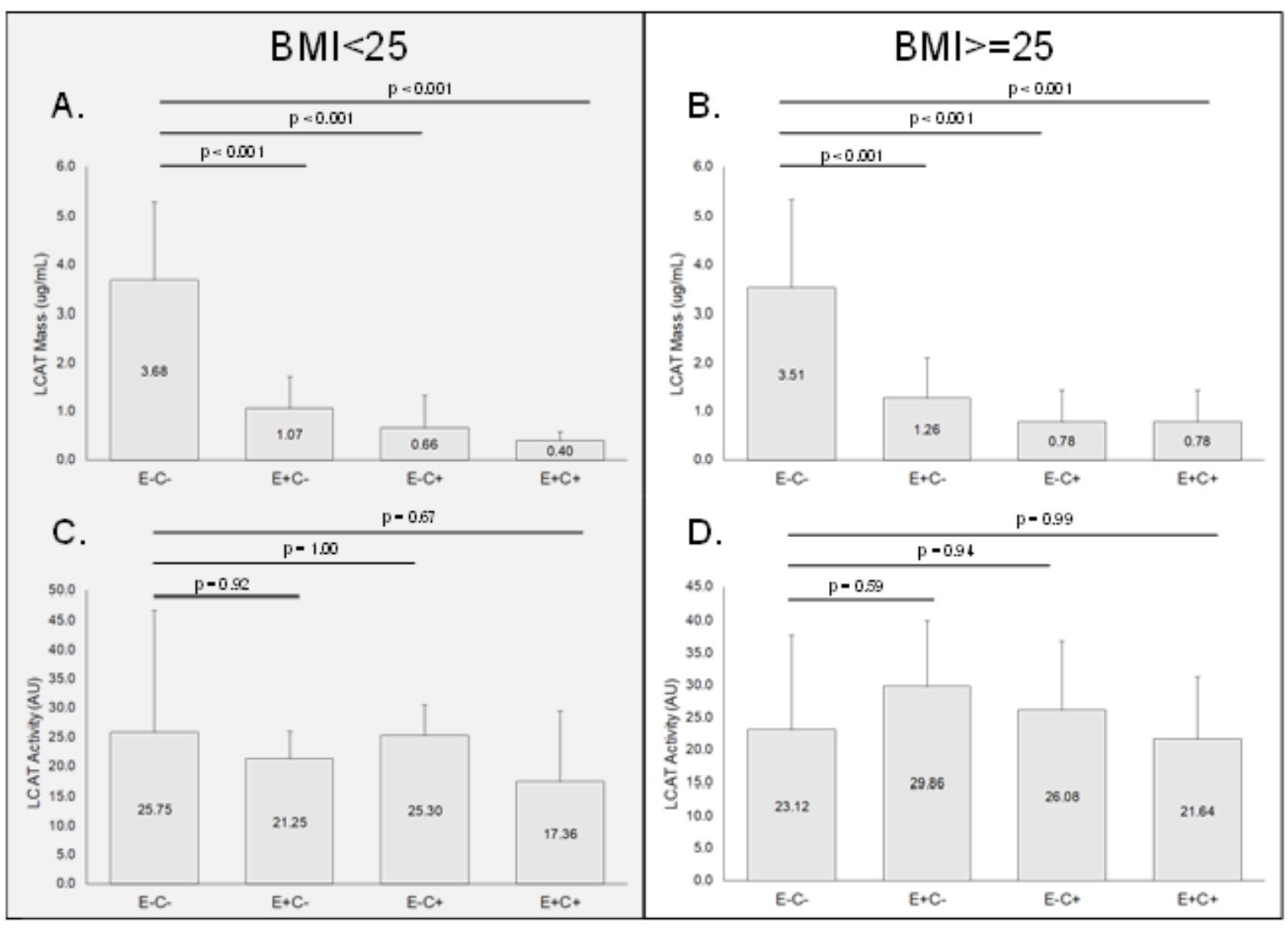

Figure 4

LCAT concentrations and activities in HDL subfractions defined by their apoE and apoC-III content, in patients with body-mass index below $25 \mathrm{Kg} / \mathrm{m} 2(\mathrm{n}=7$, left side, panels $A$ and $C$ ) or equal to or greater than $25 \mathrm{Kg} / \mathrm{m} 2$ ( $n=11$, right side, panels $B$ and $D)$. 


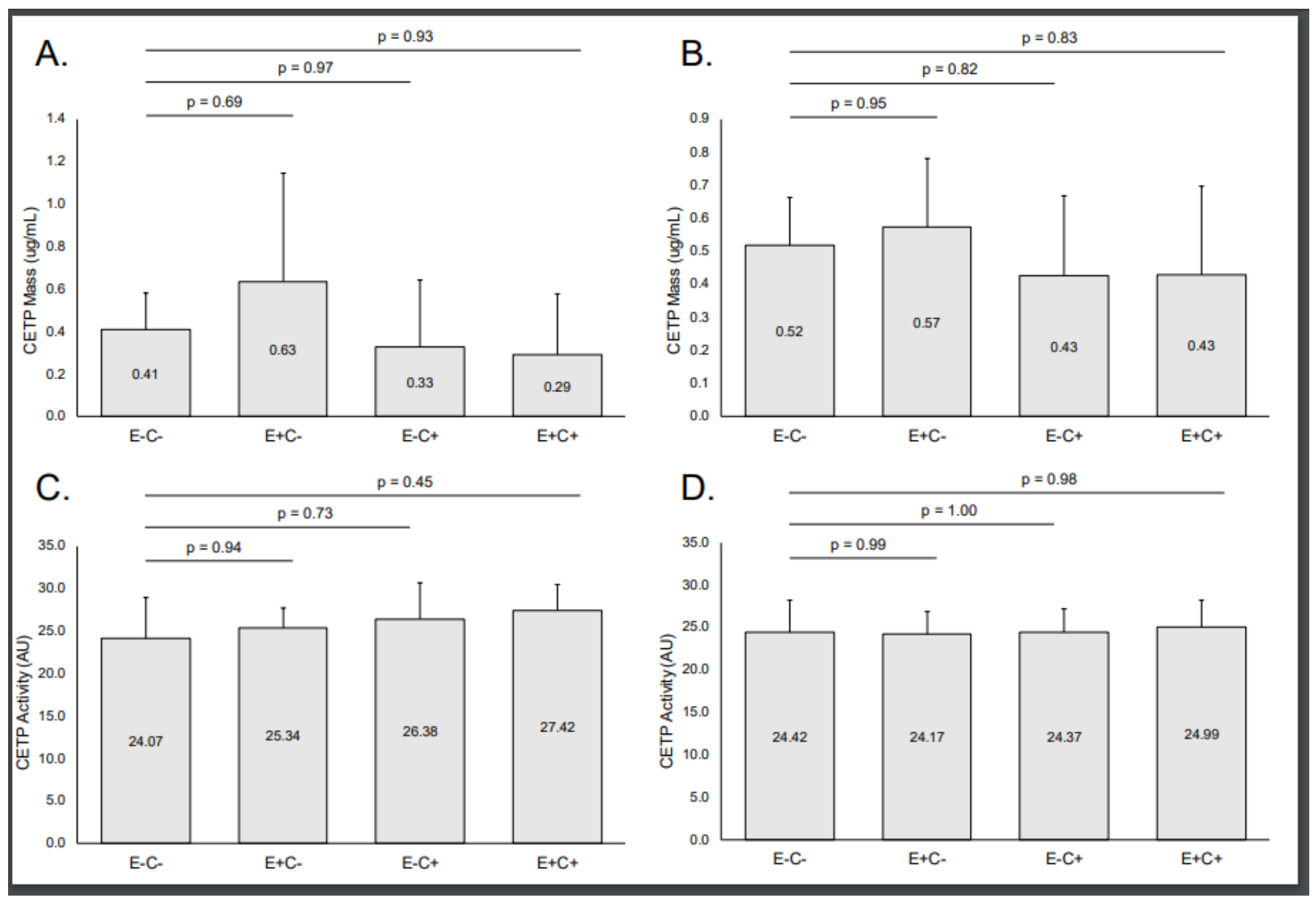

Figure 5

CETP concentrations and activities in HDL subfractions defined by their apoE and apoC-III content, in patients with body-mass index below $25 \mathrm{Kg} / \mathrm{m} 2(\mathrm{n}=7$, left side, panels $A$ and $C$ ) or equal to or greater than $25 \mathrm{Kg} / \mathrm{m} 2$ ( $\mathrm{n}=11$, right side, panels $B$ and $\mathrm{D})$. 